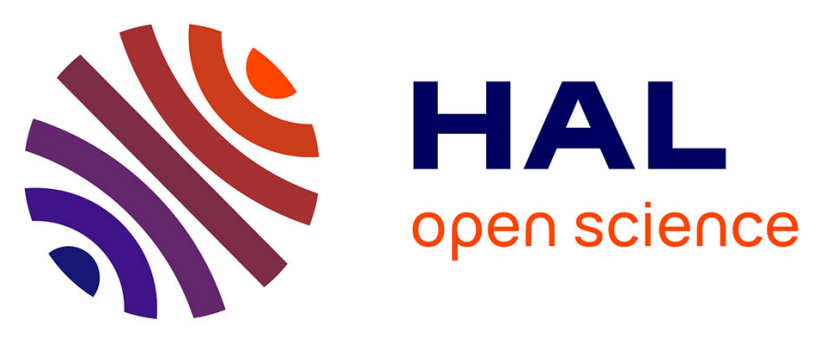

\title{
Top-Down Definition of Design Spaces Based on Skeleton Modelling
}

Nicolas Petrazoller, Frédéric Demoly, Samuel Gomes

\section{To cite this version:}

Nicolas Petrazoller, Frédéric Demoly, Samuel Gomes. Top-Down Definition of Design Spaces Based on Skeleton Modelling. IFIP International Conference on Advances in Production Management Systems (APMS), Sep 2014, Ajaccio, France. pp.249-256, 10.1007/978-3-662-44739-0_31 . hal-01388254

\section{HAL Id: hal-01388254 https://hal.inria.fr/hal-01388254}

Submitted on 26 Oct 2016

HAL is a multi-disciplinary open access archive for the deposit and dissemination of scientific research documents, whether they are published or not. The documents may come from teaching and research institutions in France or abroad, or from public or private research centers.
L'archive ouverte pluridisciplinaire HAL, est destinée au dépôt et à la diffusion de documents scientifiques de niveau recherche, publiés ou non, émanant des établissements d'enseignement et de recherche français ou étrangers, des laboratoires publics ou privés.

\section{(c)(1)}

Distributed under a Creative Commons Attribution| 4.0 International License 


\title{
Top-down definition of design spaces based on skeleton modelling
}

\author{
Nicolas Petrazoller^, Frédéric Demoly, and Samuel Gomes \\ IRTES-M3M, \\ Université de Technologie de Belfort-Montbéliard (UTBM), \\ 90010 Belfort Cedex, France
}

\begin{abstract}
This paper introduces a novel modelling approach based on the analogy to the incubator concept so as to provide a suitable support for designers through product design process. The main objective is to define a knowledge-intensive design context in the early product design stages. The main goal of the proposed approach is to provide a knowledge-based design context for designers by considering engineering knowledge in an appropriate and seamless manner. As such, the proposed design incubator will assist designers to make better-informed decisions by delivering knowledge and engineering information at the right time. A case study has been introduce to illustrate the relevance of the proposed approach.
\end{abstract}

Keywords: Assembly modelling, Skeleton-based modelling, Top-down assembly design, Proactive engineering, Design context definition, Knowledgeintensive design

\section{Introduction}

The current globalised competitive context requires industry to reduce development lead times at optimised costs and improve the quality and the efficiency of their products in order to fulfil customers' requirements. To reach these goals, a phase of architectural design is required. The role of product architects will be consider the numerous number of constraints (i.e. reliability, sustainability, ...) in the product design process. Product architects also have a global view on the system to be developed especially on functional, structural, behavioural, geometric and physical aspects which are associated to different viewpoints [6].

The fact of working collaboratively and remotely on the same product is part of a recurrent issues in large-scaled companies. As such, it is important to define a design context to assist designers through their activities. This paper is based on previous research works on proactive design for assembly and skeleton based

\footnotetext{
* Corresponding author.
} 
modelling approaches ([7] [8] [9]). Built on this, the whole is to propose a proactive top-down modelling approach of layout elements based on an analogical reasoning approach with incubator in the early product design stage. The incubator is composed of several layers of engineering information and knowledge (i.e. skeleton entities, functional surfaces, design spaces, parameters, knowledge and requirements to name a few). Compared to previous research efforts in this field [8], this approach will introduce the generation of design spaces from skeleton interface entities and functional surfaces.

Built on this, section 2 reviews some previous research works in the field of top-down and layout modelling design. In Section 3, a presentation of the design incubator concept is proposed and the overall approach is also presented. Then section 4 discusses about the deployment of the approach through a mechanical assembly. Finally, conclusions and future work are given.

\section{Related works}

This section aims to give a brief overview of published research works on topdown and layout modelling design issues, so as to provide the foundation of the proposed approach based on current status and challenges.

\subsection{Top-down design}

Top-down approach starts with the formalisation of requirements and the establishment of general layout models, specifying but not detailing any first-level components. Then, components are refined in greater details until the overall definition of the product. Support for top-down design and multiple viewpoints is a key point of top-down design which should be take into account when developing computer environments for mechanical assembly design [15]. An overview in this research domain [22] highlights some issues such as assembly model representation for top-down product design and the reasoning method from conceptual model top parametric model which must be tackled. In addition, Mäntylä [16]state that the design process could be decomposed into several stages (i.e. functional, conceptual and detailed design) while a top-down design process should support multiple abstraction models.

\subsection{Layout Modelling}

The product design process is composed of several stages from the identification of customer needs to the detailed definition of product [18]. Layout design has a crucial role to play [3] in the embodiment design stage which is the focus of this paper. By using layout elements (featured by geometric entities and engineering information), it is possible to support designers activities and exchange design data with other teams. In literature, many attempts have been made to carry out various aspects of the layout design. [12] uses layout elements to define kinematics constraints between functional components. Li et al. 
[14] capture all the feasible designs to find an optimal geometry by integrating user-defined constraints. Theodosiou et al. [20] developed full comprehensive models for spatial constraints, and in particular, for free space requirements. Ballu et al. [2] proposed a functional design method that early aides to identify and analyse functional geometrical entities and tolerance (Technologically and Topologically Related Surfaces). Mun et al. [17] proposed a solution to share skeleton model among companies working collaboratively in order to protect their intellectual property. Delgado et al. [5] uses spatial design to generate a structural solution inside it. Moreover, Gelston et al. [11] proposed a method for reconstructing boundary surfaces corresponding to skeletal curves, and then extend the method for reconstruction of boundary surfaces corresponding to skeletal surfaces. Kovacs et al. [13] describes the incremental development of a knowledge-based system for supporting floor plan design. Bai et al. [1] deployed tools based on Product Layout Feature concept to carry out collaborative design activities among multi-disciplinary teams. Skander et al. [21] proposed a skeleton-based method and models that tackle the issue of manufacturing processes selection and constraints integration, as soon as possible, into the product modelling stage. Csabai et al. [4] uses design spaces to analyse interferences between product components. Gane et al. [10] defined a methodology for building and managing requirements driven design spaces with parametric Computer Aided Design tools.

\section{Proposed approach}

This section presents the proposed approach which introduces the design incubator concept (Fig. 1). Design incubator provides a knowledge-intensive and living support to designers by defining layout product geometry at the beginning of the embodiment design phase. Such analogical reasoning will enable the introduction of a novel paradigm in CAD modelling stage.

The incubator improve coordination and information flows between three systems, such as Product Data Management (PDM) system, knowledge base and CAD system. Its role is first to generate a design context to designers. Then, when designers start their activities, it will identify and analyse their intents. Once design intents are captured, a request to the knowledge base is process in order to get appropriate engineering information or knowledge for designers at the right time. Finally, when designers store their component, the incubator will generate design intents history. Such procedures will ensure understanding and traceability in design with new technical objects.

\subsection{Overall methodology description}

Based on the SKL-ACD approach[7] [19], an enriched flow chart is introduced to describe the proposed approach in a more detailed view (Fig. 2). An explanation of the different steps of the method is visible below: 


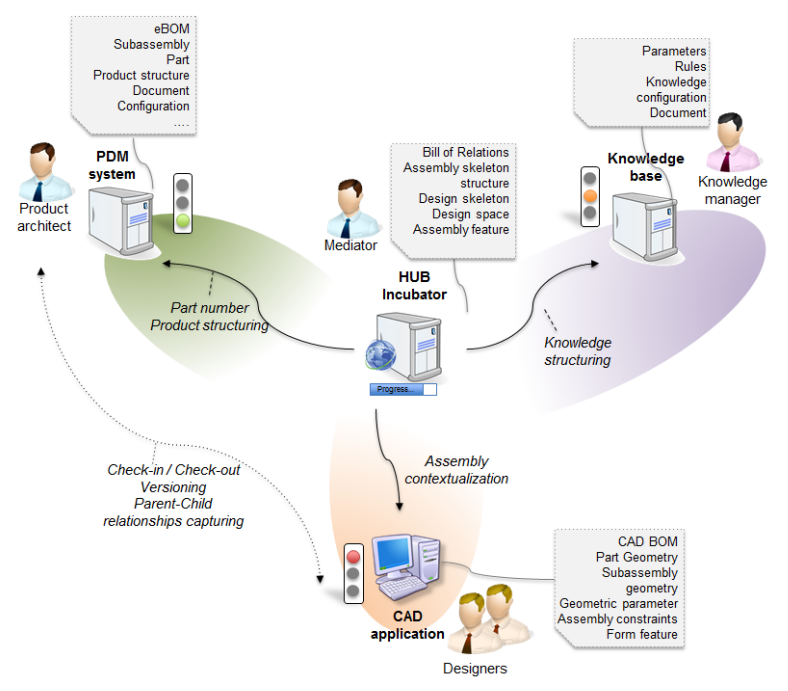

Fig. 1. Incubator concept.

- Start: Starting from the early defined assembly sequence and product relational information embedded in graphs and matrices, the product architect defines kinematics/technological pairs in the directed graph;

- Steps 1 and 2. The product structure is automatically generated and assembly skeleton places are assigned inside (Steps 1 and 2 of Fig. 2);

- Steps 3 and 4. Based on these relationships, assembly constraints are automatically defined and geometric skeleton entities are generated in order to provide interface control elements for assembly modelling (Steps 3 and 4 of Fig. 2);

- Step 5. The product architect introduces new assembly constraints between the generated geometric skeleton entities (Step 5 of Fig. 2); A new graph, called skeleton graph, built upon these constraints, is defined by skeleton entities and their related assembly constraints;

- Step 6. This graph is simplified later on by the generalization and the concatenation of skeleton elements into a minimal skeleton graph (Step 6 of Fig. 2);

- Step 7. Based on this minimal skeleton graph and the early-defined assembly sequence, this step allows the structuring and regrouping of skeleton elements in assembly skeletons (Step 7 of Fig. 2);

- Step 8. An assembly coordinate system is defined for each assembly layer and associated to each identified base part. Therefore new constraints are introduced to link the defined assembly coordinate systems with the interface control elements from the minimal skeleton graph (Step 8 of Fig. 2);

- Step 2. The resulting assembly skeletons can be allocated to the initial product structure. At this stage, it is possible to assign rights to a skeleton entity which is at the interface of different assembly skeletons (Step 2 of Fig. 2); 
- Step 9. Based on the kinematic pairs between components and product architect choice, the skeleton interfaces entities are defined (Step 9 of Fig. 2);

- Step 10. Supported by skeleton interfaces entities, functional surfaces are defined (Step 10 of Fig. 2);

- Step 11. From functional surfaces and skeleton interface entities, design spaces are generated (Step 11 of Fig. 2);

- End: As a result, the assembly skeleton CAD model, functional surfaces and design spaces are semi-automatically generated.

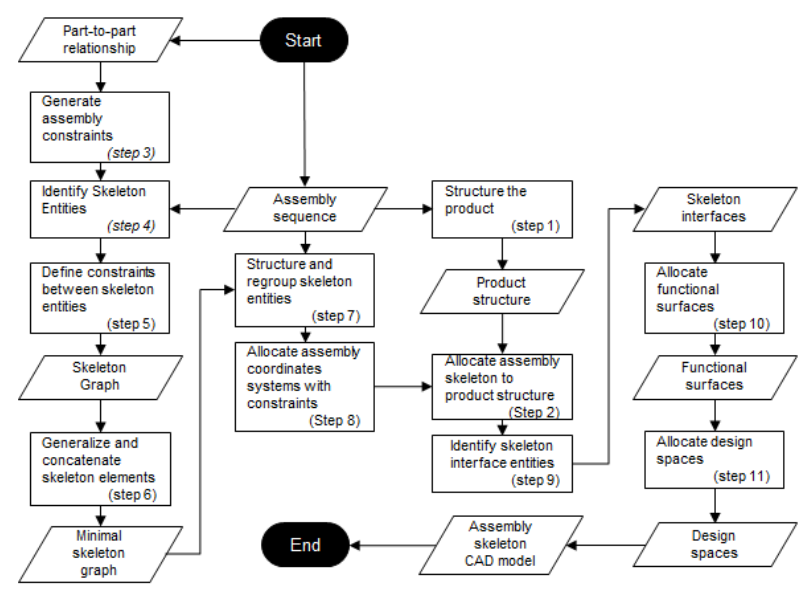

Fig. 2. Enriched flowchart of the proposed approach.

\section{Case Study}

In this section, the proposed approach is illustrated with a mechanical system (Fig. 3). Each step of the method are described for a better understanding (Fig. 4 and Fig. 5).

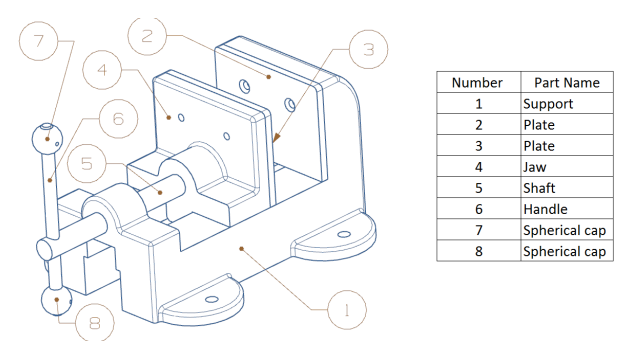

Fig. 3. Case study : vice and parts list of the case study. 
4.1 Determination of the skeleton minimal graph (Step 3 to 6 of Fig. 2) and Introduction of an assembly coordinate system (Step 7 to 8 of Fig. 2)

The product architect starts by defining the kinematic pairs between each parts. Based on the kinematic pairs, the skeleton entities and position constraints between each parts are deducted. At this point, to facilitate the management of the skeleton entities, it is possible to simplify the proposed skeleton graph by generalize and concatenate skeleton entities. Based on the minimal skeleton graph of the previous step, an assembly coordinate system is introduced. Each skeleton entities are linked to this new assembly coordinate system by the intermediate of geometrical constraints. Then, the geometrical constraints are concatenated.

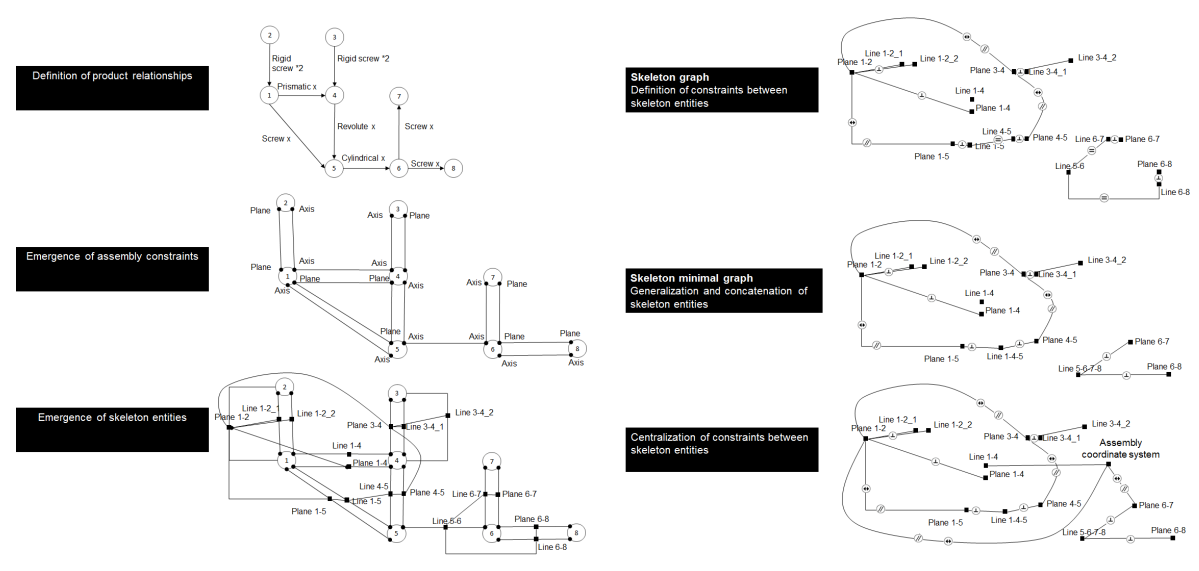

Fig. 4. Definition process of a minimal skeleton model.

\subsection{Allocation of skeleton interface entities and functional surfaces (step 9 to 10 of Fig. 2) and generation of design spaces (step 11 of Fig. 2)}

Based on previous steps, the skeleton interface entities are defined and associated to skeleton entities.

Then, it is possible to concatenate skeleton interface entities in order to simplify the design environment. Finally, after this simplification, the functional surface are deduced from the skeleton interface entities. 


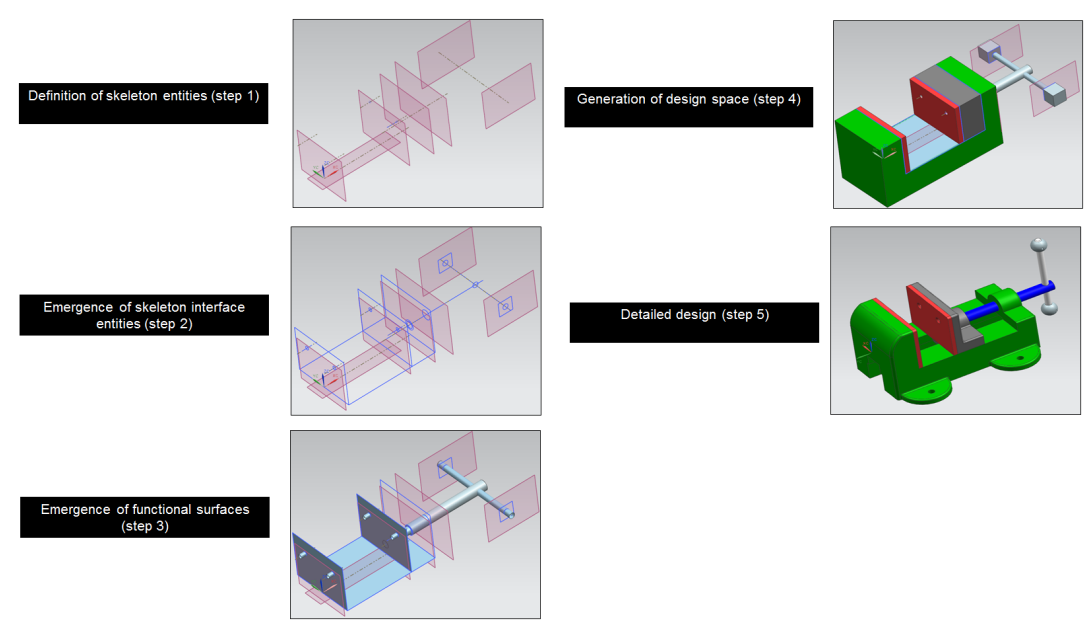

Fig. 5. Definition process of design spaces models.

\section{Conclusions and future work}

In this paper, a novel approach to define a design context in the early phases of the design development has been proposed and described in detail. The current issue of the paper is to allocate design spaces to the assembly skeleton model. Defining design spaces based on skeleton modelling will permit a better understanding of "what to design" by designers. To illustrate the feasibility and the relevance of the proposed approach, a use case has been carried out. Finally, three main issues demand further research: the incorporation of knowledge; adding requirements; and create a link between the method and PDM system.

\section{Acknowledgements}

The research activity is part of the INGéPROD (Productiveness for ProductProcess Engineering in a Design Chain context), which has been funded by French Automotive Cluster Pôle de Compétitivité Véhicule du Futur. The authors would like to thank General Electric for this collaboration and all the financial supports of this research and technology program: DRIRE de FrancheComté, Communauté dAgglomération du Pays de Montbéliard, Conseil Général du Doubs and Conseil Régional de Franche-Comté.

\section{References}

1. Bai, Y.W., Chen, Z.N., Bin, H.Z., Hu, J.: Collaborative design in product development based on product layout model, Robotics and Computer-Integrated Manufacturing 21 (2005) 55-65 
2. Ballu,A., Falgarone,H., Chevassus, N., Mathieu,L.: New Design Method based on Functions and Tolerance Specifications for Product Modelling, Annals of the CIRP

3. Chen, X., Gao, S., Yang, Y., Zhang, S.: Multi-level assembly model for top-down design of mechanical products, Computer-Aided Design, 44 (2012) 1033-1048

4. Csabai, A., Stroud, I., Xirouchakis, P.C.: Container spaces and functional features for top-down 3D layout design, Computer-Aided Design 34 (2002) 1011-1035

5. Davila Delgado,J.M., Hofmeyer,H.: Automated generation of structural solutions based on spatial designs, Automation in Construction, 35 (2013), 528-541

6. Demoly, F., Monticolo, D., Eynard, B., Rivest, L., Gomes, S.: Multiple viewpoint modelling framework enabling integrated productprocess design. International Journal on Interactive Design and Manufacturing, 4 (2010) 269-280

7. Demoly, F., Yan, X.-T., Eynard, B., Rivest, L., Gomes, S.: An Assembly oriented design framework for product structure engineering and assembly sequence planning. Robotics and Computer-Integrated Manufacturing, 27 (2011) 33-46

8. Demoly, F., Toussaint, L., Eynard, B., Kiritsis, D., Gomes, S.: Geometric skeleton computation enabling concurrent product engineering and assembly sequence planning. Computer-Aided Design, 43 (2011) 1654-1673

9. Demoly, F., Yan, X.-T., Eynard, B., Kiritsis, D., Gomes, S.: Integrated product relationships management: a model to enable concurrent product design and assembly sequence planning. Journal of Engineering Design, 23 (2012) 544-561

10. Gane, V., Haymaker, J.: Design Scenarios: Enabling transparent parametric design spaces, 6 (2012) 618-640

11. Gelston,S.M., Dutta,D.: Boundary surface recovery from skeleton curves and surfaces, Computer Aided Geometric Design, 12 (1995), 27-51

12. Kim, K.J., Sacks, E., Joskowicz, L.: Kinematic analysis of spatial fixed-axis higher pairs using configuration space, 6 (2001) 279-291

13. Kovfics,L.B.: Knowledge based floor plan design by space partitioning: A logic programming approach, Artificial Intelligence in Engineering, 6 (1991), 162-185

14. Li, C.G., Li, C.L, Liu, Y., Huang, Y.: A new C-space method to automate the layout design of injection mould cooling system, 6 (2012) 811-823

15. Libardi E., Dixon J., Simmons M.: Computer environments for the design of mechanical assemblies: a research review, Engineering with Computers, 3 (1988)

16. Mntyl M.: A modeling system for top-down design of assembled products, IBM Journal of Research and Development, 34 (1990), 636-659

17. Mun,D., Hwang,J., Han,S.: Protection of intellectual property based on a skeleton model in product design collaboration, Computer-Aided Design, 41 (2009), 641-648

18. Pahl, G., Beitz, W.: Engineering design, a systematic approach, 2nd ed. London: Springer, (1996) 544

19. Petrazoller, N., Demoly, F., Deniaud, S., Gomes, S., Towards a knowledge-intensive framework for top-down design context definition. APMS 2013 conference, (2013)

20. Theodosiou, G., Sapidis, N.S.: Information of layout constraints for product lifecycle management: a solid-modelling approach, 6 (2003) 549-564

21. Skander,A., Roucoules,L., Klein Meyer,J.S.: Design and manufacturing interface modelling for manufacturing processes selection and knowledge synthesis in design, International Journal of Advanced Manufacturing Technology, 37 (2008), 443-454

22. Wen Jian L., Tian guo J.: Research state and development directions of product top-down design., Computer Integrated Manufacturing Systems, 2002 